\title{
56 Endurance training and fatty tissue
}

The key benefits of physical activity and exercise are:

- Breaking down excessive fatty tissue with significant benefits on metabolism and the cardiovascular system

- Reducing the incidence of chronic diseases

- General reduction in body weight, which in turn relieves pressure on the joints - all associated with enhanced psychological well-being

Regular exercise lowers triglyceride levels. When fatty tissue is broken down, abdominal fat is reduced first. This has a positive effect given that abdominal fatty tissue is particularly damaging to health ( $\triangleright$ Chapter 38 and $\triangleright$ Chapter 39 ).

Cholesterol levels change very little as a result of physical activity. The findings of numerous randomized, controlled studies show a slight increase in HDL cholesterol levels and a slight decrease in LDL cholesterol levels. The LDL particles increase in size as a result of endurance sport, which helps to reduce the risk of atherosclerosis as small LDL particles carry a higher risk ( $\downarrow$ Chapter 18 ).

The majority of all hip and knee replacements can be linked to overweight and obesity (Anandacoomarasamy et al. 2009, Wang et al. 2009, Smith et al. 2014).

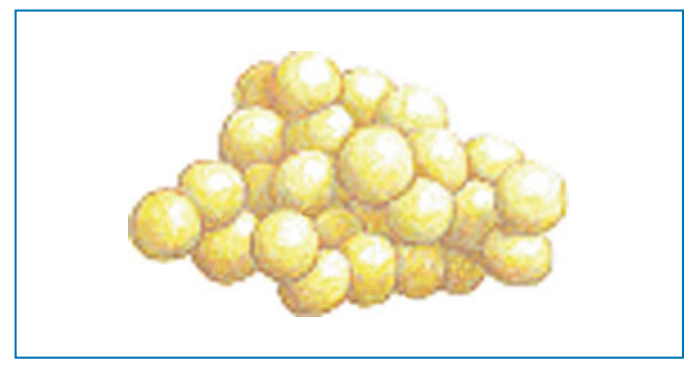

- Fig. 56.1

The aesthetic effect should not be underestimated either. The reduction in waistline resulting from more exercise considerably reinforces people's general self-esteem.

It is not just the excess weight that is responsible for the joint damage but also the hormone leptin produced by the fatty tissue ( $\triangleright$ Chapter 13 ). This can damage the joint cartilage by causing chronic lowgrade inflammation. The breakdown of fatty tissue caused by endurance training therefore has a twofold benefit for the joints. There is less weight on them and less leptin to damage them. 